\title{
Self-rated health in university students: a systematic review
}

MARIANA DA Silva Ferreira $^{I}$ Gildeene Silva Farias ${ }^{\text {II }}$ Sandra Adriana Neves Nunes ${ }^{\text {III }}$ Camila Bosquiero Papini ${ }^{\text {IV }}$ Thiago Ferreira de Sousa ${ }^{v}$ 'Secretaria Municipal de Educação de Teresina, Teresina/PI - Brasil "Faculdade Estácio de Teresina, Teresina/PI - Brasil

IIIUniversidade Federal do Sul da Bahia (UFSB), Itabuna/BA - Brasil IVUniversidade Federal do Triângulo Mineiro (UFTM), Uberaba/MG Brasil

vUniversidade Federal do Recôncavo da Bahia (UFRB), Amargosa/ BA - Brasil

\begin{abstract}
Objective: To determine, through a systematic review, the prevalence of self-rated health (HSE) in university students, as well as the factors associated. Methods: The data was collected in the databases PubMed, SciELO, Virtual Health Library, LILACS, Web of Science and Scopus, and through manual searches. This study adopted the PRISMA checklist and recommendations. The selected articles were analyzed according to their methodological characteristics and the critical evaluation guidelines. Studies that estimated HSE in university students were eligible. Results: Of the 1.416 articles found, 19 composed this review. Positive results in the HSE varied from $61.8 \%$ to $94.5 \%$, and negative HSE varied from $3.4 \%$ to $38.2 \%$, in students from different countries. The cross-sectional design was adopted in all studies. The studies used different questions and answers in the HSE. Having inadequate nutrition, leisure-time physical inactivity, severe stress, and health complaints were the factors that were most associated with a negative HSE. Conclusions: The prevalence results of negative SRH in university students from different countries and the associated factors indicate the need for health promotion actions in this environment.
\end{abstract}

Keywords: Health Status, Students, Cross-Sectional Studies.

Resumo - Objetivo: O objetivo foi determinar, por meio de uma revisão sistemática, as prevalências da autoavaliação de saúde (AAS) em universitários, bem como os fatores a ela associados. Métodos: As buscas dos estudos foram realizadas nas bases de dados PubMed, SciELO, Biblioteca Virtual em Saúde, LILACS, Web of Science e Scopus, além de buscas manuais. Este estudo adotou os itens e recomendações do PRISMA e os artigos selecionados foram analisados conforme as características metodológicas e as diretrizes de avaliação crítica. Foram elegíveis estudos que estimaram a AAS em universitários. Resultados: Dos 1.416 artigos encontrados nas bases de dados, 19 fizeram parte dessa revisão. Foram observadas prevalências para a AAS positiva de $61,8 \%$ a $94,5 \%$ e para a AAS negativa foram de $3,4 \%$ a $38,2 \%$ em universitários de diferentes países. O desenho de pesquisa adotado em todos os estudos foi o transversal. Entre os estudos houve a aplicação de diferentes perguntas e opções de resposta para a AAS. Observou-se que ter alimentação inadequada, inatividade física no lazer, estresse elevado e ter queixas de saúde foram os fatores que mais se associaram a AAS negativa. Conclusão: A amplitude na prevalência da AAS negativa em universitários de diferentes países e os fatores associados indicam a necessidade de ações de promoção de saúde no ambiente universitário.

Palavras-Chave: Nível de Saúde, Estudantes, Estudos TransVERSAIS. 


\section{INTRODUCTION}

Health self-evaluations (HSE) are measures used in epidemiological surveys $s^{1,2}$ and are quantified using a single question, which has high validity and reliability ${ }^{1}$. The World Health Organization (WHO) ${ }^{3}$ recommends these indicators to estimate the health level in population-based studies and it has been used in studies with children ${ }^{4}$, adolescents ${ }^{5}$, adults $^{6}$ or the elderly ${ }^{7}$.

Evidence suggests that this item is predictive in relation to morbidity and mortality outcomes ${ }^{8,9}$. These outcomes also suffer influences from attributes that are associated with this measure, such as: sociodemographic variables (women, old age, lower income and educational level $)^{10}$ and health-related behaviors (insufficiently active, irregular consumption of fruits and vegetables, excessive drinking and smoking $)^{10,11}$ and multiple biomarker levels (C-reactive protein, HDL cholesterol, glycosylated hemoglobin, 25-hydroxyl-vitamin-D, zeaxanthin, apolipoprotein-B, cell-free DNA and protein carbonyls ${ }^{12}$.

Among the general population, university students are more susceptible to the impact of academic life on their health status, due to being subject to stressful situations in the activities required by the academic context ${ }^{13}$, in addition to the adoption of health-risk behaviors during this period ${ }^{14}$. Therefore, it is essential to perform investigations about the perception of health in this specific public. Especially due to the process of transition from adolescence to adulthood and the possible adoption of negative health behaviors $^{15,16}$, which can be decisive on the results of perceived health.

The studies on HSE in university students, it was observed that being a woman, having a longer time of exposure to the university environment, having lower inco- $m e^{17-19}$, being inactive during leisure time, having inadequate nutrition, experiencing negative stress in life, and consuming alcoholic beverages ${ }^{19-21}$ are factors that are associated with negative HSE. However, considering the relevance of assessing the HSE in this group, a scarcity or lack of review studies was noticed in the indexed databases.

It is expected that, with the knowledge of the health status, as well as the evidence about the factors that are associated with HSE in university students, it will be possible to contribute to the characterization of the components that need to be strengthened and monitored over time. In addition, the information in this review may support targeted projects or programs focusing on the health of this group. This study aimed to determine, through a systematic review, the prevalences found by HSEs in university students, as well as the associated factors.

\section{Methods}

This is a systematic review, which adopted the Preferred Reporting Items for Systematic reviews and Meta-Analyzes (PRIS$\mathrm{MA})^{22}$ methodology. The proposal for this review was registered in the International Prospective Register of Systematic Reviews (PROSPERO - CRD 42019128446), under the title "Self-evaluation of health in university studies: a systematic review".

\section{Search}

The advanced search for articles on the topic was carried out in the English language, in the electronic databases: National Library of Medicine (PubMed), Scientific Electronic Library Online (SciELO), Virtual Health Library (VHL), LILACS (Latin American Literature in Sciences of the Health), Web of Science (Web of Knowledge), and Scopus, in 
April 2019. The search strategy included the keywords: "health self-assessment", "perceived health", "self-perception of health", "self-reported health", "self-evaluation of health", "health status", "self-rated health", and also "undergraduate", "college students", "academics", "university student", "university", "cross-sectional study" and "longitudinal study". The Boolean operators "AND" and "OR" were used for combinations between groups of key words, and the symbol (" ") was used for variations of composed terms.

\section{Study selection and appraisal}

To be included in this systematic review, the studies needed to meet the following criteria: a) being observational, cross-sectional or longitudinal studies; b) having HSE as a dependent variable; c) being in English, Portuguese or Spanish; d) having as a population university students without age restriction; e) being original studies. There was no limitation on the year of publication.

The initial selection was based on the titles of the articles. After this identification, the abstracts were read. After this stage, the articles chosen were read in full and, subsequently, selected according to the inclusion criteria. Manual searches were performed considering the references of the selected articles in order to find possible studies that met the selection criteria, regardless of the publication database. In addition, a search of publications was carried out in the Google Scholar database, with the following Portuguese keywords: "autoavaliação de saúde", "percepção de saúde", "universitários" and "acadêmicos". These steps were carried out independently and conducted by two reviewers, and a third helped when there were disagreements. The Zotero software was used to store the articles and exclude duplicates, and the Excel software was used to tabulate the extracted data.
The information extracted from the articles that met the inclusion criteria were: a) author; b) year of publication; c) impact factor of the journal; d) study design; e) country where the study was carried out; f) age range of participants; g) gender; h) course or study area; i) sample; j) sample selection criteria; k) question to identify the dependent variable; 1 ) HSE response options; $m$ ) prevalence found by the HSE; $n$ ) variables associated with the outcome; and o) association test (comparison) used in the main analyses.

\section{Study-quality appraisal}

The quality of each article was judged based on the methodological considerations of the Assessing Cardiovascular Risk: Systematic Evidence Review, From the Risk Assessment Work Group ${ }^{23}$ for observational cohort research and cross-sectional studies. This instrument has 14 items of quality assessment, which include the objective of the study; its definition, selection, composition and participation of the studied population; the definition and evaluation of exposure and outcome variables; the amount of exposure before the result; monitoring the study and adjusting confounding variables. Each item was given "yes" if the article presented the expected answer and "no" when it did not answer the item's question. If the question did not fit the article, it would have as answer options: cannot be determined (CD), not reported (NR) and not applicable (NA). The quality analysis of the study (good, regular or poor) was defined according to two reviewers.

\section{RESULTS}

The search for studies that identified HSE results in university students resulted in 1.416 articles, 399 of which were duplicates. After excluding studies that did not meet the 
initial criteria, 27 studies were read in full. Of these, six articles did not present the prevalences found by the HSE as outcomes, one (1) was not an original article, one (1) was from a language not considered in this review, and in eight publications the instrument assessed health-related quality of life. Thus, 11 arti- cles $^{18,20,21,24-31}$ met the pre-established inclusion criteria. After this phase, a manual search was performed, where six articles ${ }^{17,19,32-35}$ cited in the references of the 11 selected articles and two articles ${ }^{36,37}$ from another database (Google Scholar) were included in this review, totaling 19 articles (Figure 1).

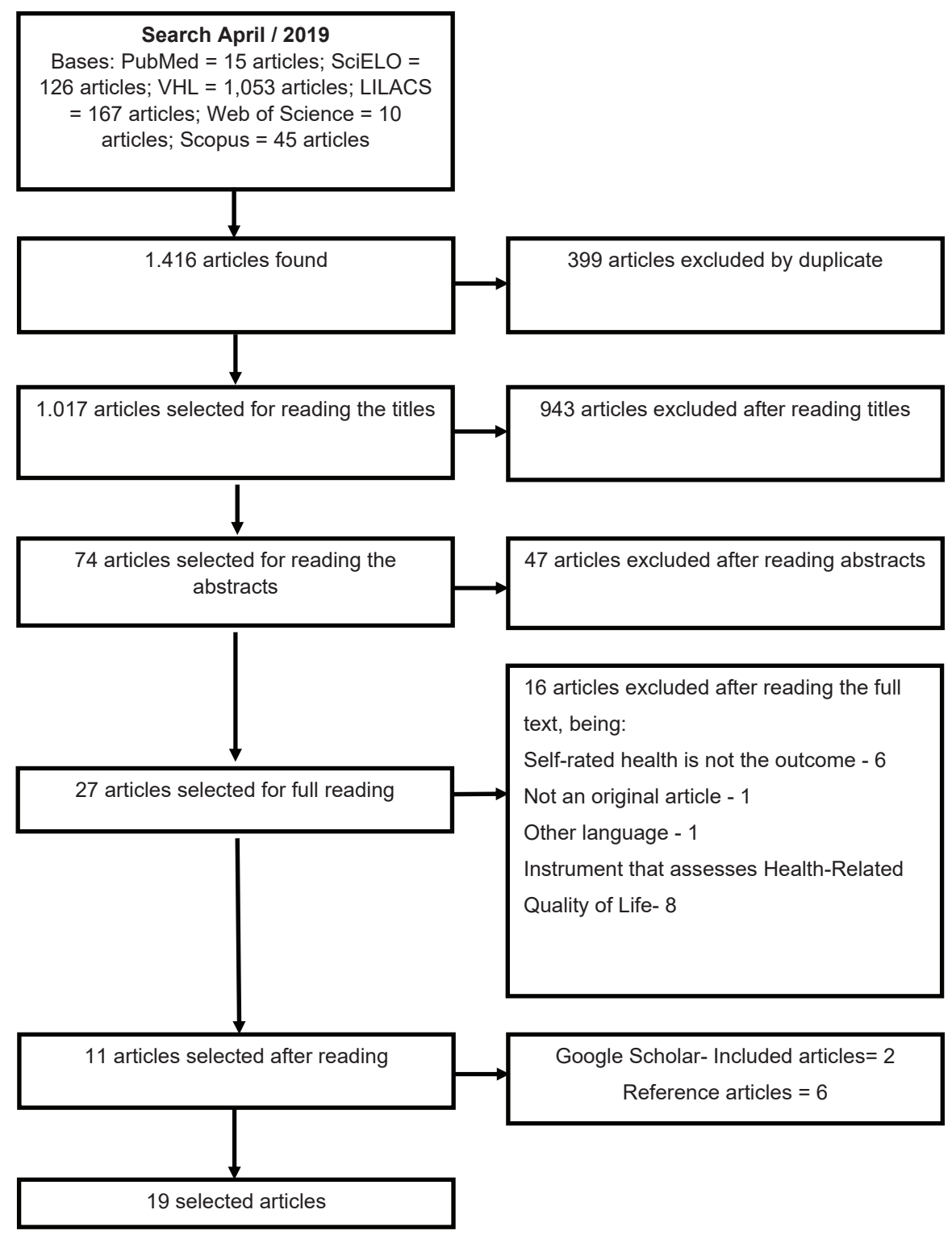

Figure 1 - Flowchart of the study selection process for this systematic review. 
Table 1 shows that among the 19 studies, only a small number of publications met the quality criteria for the outcome assessment methods. It was observed that $36.84 \%(n=7)$ of the articles presented a "good" classification, eight articles were "regular", and four articles were "bad".

Table 1. Guidelines used for critical evaluation of publications about self-rated health studies in university students. Evaluation criteria proposed by Assessing Cardiovascular Risk: Systematic

Evidence Review from the Risk Assessment Work Group ${ }^{23}$.

\begin{tabular}{|c|c|}
\hline Criteria & $\mathbf{n} / \mathbf{N}(\%)$ \\
\hline $\begin{array}{l}\text { 1. Is the research question or objective clearly stated in this } \\
\text { study? }\end{array}$ & $19 / 19(100 \%)$ \\
\hline 2. Is the study population clearly specified and defined? & $16 / 19(84.21 \%)$ \\
\hline 3. Is the participation rate of eligible people at least $50 \%$ ? & $14 / 19(73.68 \%)$ \\
\hline 4. Were all participants selected or recruited from the same & \\
\hline $\begin{array}{l}\text { or similar population (including the same time period)? } \\
\text { Were the inclusion and exclusion criteria pre-established } \\
\text { and applied uniformly to all participants? }\end{array}$ & $18 / 19(94.73 \%)$ \\
\hline $\begin{array}{l}\text { 5. Were justifications for sample size, description of power } \\
\text { or estimates of variation and effect provided? }\end{array}$ & $11 / 19(57.89 \%)$ \\
\hline $\begin{array}{l}\text { 6. For the analyzes in this study, were the exposures of } \\
\text { interest measured before the outcomes? }\end{array}$ & $0 / 19(0.00 \%)$ \\
\hline $\begin{array}{l}\text { 7. Was the period of time sufficient for the individual to } \\
\text { reasonably be able to see an association between exposure } \\
\text { and outcome? }\end{array}$ & $0 / 19(0.00 \%)$ \\
\hline $\begin{array}{l}\text { 8. For exposures that may vary in quantity or level, did the } \\
\text { study examine different levels of exposure related to the } \\
\text { outcome (for example, categories of exposure, or exposure } \\
\text { measured as a continuous variable)? }\end{array}$ & $3 / 19(15.78 \%)$ \\
\hline $\begin{array}{l}\text { 9. Were exposure measures (independent variables) clearly } \\
\text { defined, validated, reliable and implemented consistently } \\
\text { among all study participants? }\end{array}$ & $19 / 19(100 \%)$ \\
\hline $\begin{array}{l}\text { 10. Have exposures been evaluated more than once over } \\
\text { time? }\end{array}$ & $0 / 19(0.00 \%)$ \\
\hline $\begin{array}{l}\text { 11. Were the outcome measures (dependent variables) } \\
\text { clearly defined, validated, reliable and implemented } \\
\text { consistently among all study participants? }\end{array}$ & $19 / 19(100 \%)$ \\
\hline $\begin{array}{l}\text { 12. Were the outcome evaluators blinded to the } \\
\text { participants' exposure condition? }\end{array}$ & $0 / 19(0.00 \%)$ \\
\hline $\begin{array}{l}\text { 13. Was the loss of follow-up after the baseline of } 20 \% \text { or } \\
\text { less? }\end{array}$ & $0 / 19(0.00 \%)$ \\
\hline $\begin{array}{l}\text { 14. Were the main potential confounding variables } \\
\text { measured and adjusted statistically for their impact on the } \\
\text { relationship between exposures and outcomes? }\end{array}$ & $13 / 19(68.42 \%)$ \\
\hline
\end{tabular}




\begin{tabular}{|c|c|c|c|c|c|c|c|c|c|c|c|c|c|c|c|}
\hline \multirow{2}{*}{ Selected Articles } & \multicolumn{15}{|c|}{ Assessed items } \\
\hline & 1 & 2 & 3 & 4 & 5 & 6 & 7 & 8 & 9 & 10 & 11 & 12 & 13 & 14 & Evaluation \\
\hline 1.Castrén et al. ${ }^{26}$ & $\mathrm{Y}$ & $\mathrm{Y}$ & $\mathrm{Y}$ & $\mathrm{Y}$ & $\mathrm{Y}$ & $\mathrm{N}$ & $\mathrm{N}$ & N/A & $\mathrm{Y}$ & $\mathrm{N}$ & $\mathrm{Y}$ & $\mathrm{N}$ & N/A & $\mathrm{N}$ & REGULAR \\
\hline $\begin{array}{l}\text { 2.Mikolajczyk et } \\
\text { al. }{ }^{18}\end{array}$ & $\mathrm{Y}$ & $\mathrm{Y}$ & $\mathrm{Y}$ & $\mathrm{Y}$ & $\mathrm{N}$ & $\mathrm{N}$ & $\mathrm{N}$ & $\mathrm{Y}$ & $\mathrm{Y}$ & $\mathrm{N}$ & $\mathrm{Y}$ & $\mathrm{N}$ & N/A & $\mathrm{Y}$ & GOOD \\
\hline 3.Sousa ${ }^{17}$ & $\mathrm{Y}$ & $\mathrm{Y}$ & $\mathrm{Y}$ & $\mathrm{Y}$ & $\mathrm{Y}$ & $\mathrm{N}$ & $\mathrm{N}$ & N/A & $\mathrm{Y}$ & $\mathrm{N}$ & $\mathrm{Y}$ & $\mathrm{N}$ & N/A & $\mathrm{N}$ & REGULAR \\
\hline 4.Sousa ${ }^{32}$ & $\mathrm{Y}$ & $\mathrm{Y}$ & $\mathrm{Y}$ & $\mathrm{Y}$ & $\mathrm{Y}$ & $\mathrm{N}$ & $\mathrm{N}$ & N/A & $\mathrm{Y}$ & $\mathrm{N}$ & $\mathrm{Y}$ & $\mathrm{N}$ & N/A & $\mathrm{N}$ & REGULAR \\
\hline 5.Silva ${ }^{25}$ & $\mathrm{Y}$ & $\mathrm{Y}$ & $\mathrm{Y}$ & $\mathrm{Y}$ & $\mathrm{Y}$ & $\mathrm{N}$ & $\mathrm{N}$ & N/A & $\mathrm{Y}$ & $\mathrm{N}$ & $\mathrm{Y}$ & $\mathrm{N}$ & N/A & $\mathrm{Y}$ & GOOD \\
\hline 6.Schmidt ${ }^{24}$ & $\mathrm{Y}$ & $\mathrm{Y}$ & $\mathrm{N}$ & Y & $\mathrm{N}$ & $\mathrm{N}$ & $\mathrm{N}$ & $\mathrm{Y}$ & $\mathrm{Y}$ & $\mathrm{N}$ & Y & $\mathrm{N}$ & N/A & Y & POOR \\
\hline 7.Rohrer et al. ${ }^{27}$ & $\mathrm{Y}$ & $\mathrm{Y}$ & $\mathrm{N}$ & $\mathrm{Y}$ & $\mathrm{N}$ & $\mathrm{N}$ & $\mathrm{N}$ & N/A & $\mathrm{Y}$ & $\mathrm{N}$ & $\mathrm{Y}$ & $\mathrm{N}$ & N/A & $\mathrm{Y}$ & REGULAR \\
\hline 8.Freire et al. ${ }^{19}$ & $\mathrm{Y}$ & $\mathrm{Y}$ & $\mathrm{Y}$ & $\mathrm{Y}$ & $\mathrm{Y}$ & $\mathrm{N}$ & $\mathrm{N}$ & N/A & $\mathrm{Y}$ & $\mathrm{N}$ & $\mathrm{Y}$ & $\mathrm{N}$ & N/A & Y & GOOD \\
\hline 9.Sousa et al. ${ }^{20}$ & $\mathrm{Y}$ & $\mathrm{Y}$ & $\mathrm{Y}$ & Y & $\mathrm{Y}$ & $\mathrm{N}$ & $\mathrm{N}$ & N/A & Y & $\mathrm{N}$ & Y & $\mathrm{N}$ & N/A & $\mathrm{Y}$ & GOOD \\
\hline 10.Pengpid et al. ${ }^{33}$ & $\mathrm{Y}$ & $\mathrm{N}$ & $\mathrm{Y}$ & $\mathrm{Y}$ & $\mathrm{N}$ & $\mathrm{N}$ & $\mathrm{N}$ & N/A & $\mathrm{Y}$ & $\mathrm{N}$ & $\mathrm{Y}$ & $\mathrm{N}$ & N/A & $\mathrm{Y}$ & REGULAR \\
\hline $\begin{array}{l}\text { 11.El-Ansari e } \\
\text { Stock }^{34}\end{array}$ & $\mathrm{Y}$ & $\mathrm{N}$ & $\mathrm{N}$ & $\mathrm{Y}$ & $\mathrm{N}$ & $\mathrm{N}$ & $\mathrm{N}$ & N/A & $\mathrm{Y}$ & $\mathrm{N}$ & $\mathrm{Y}$ & $\mathrm{N}$ & N/A & $\mathrm{Y}$ & POOR \\
\hline $\begin{array}{l}\text { 12. Mikolajczyk et } \\
\text { al. }{ }^{21}\end{array}$ & $\mathrm{Y}$ & $\mathrm{Y}$ & $\mathrm{Y}$ & $\mathrm{Y}$ & $\mathrm{N}$ & $\mathrm{N}$ & $\mathrm{N}$ & N/A & $\mathrm{Y}$ & $\mathrm{N}$ & $\mathrm{Y}$ & $\mathrm{N}$ & N/A & $\mathrm{Y}$ & GOOD \\
\hline $\begin{array}{l}\text { 13.El-Ansari e } \\
\text { Stock }^{35}\end{array}$ & $\mathrm{Y}$ & $\mathrm{N}$ & $\mathrm{N} / \mathrm{R}$ & $\mathrm{N}$ & $\mathrm{N}$ & $\mathrm{N}$ & $\mathrm{N}$ & N/A & $\mathrm{Y}$ & $\mathrm{N}$ & $\mathrm{Y}$ & $\mathrm{N}$ & N/A & $\mathrm{Y}$ & POOR \\
\hline 14.Andrade et al. ${ }^{37}$ & $\mathrm{Y}$ & $\mathrm{Y}$ & $\mathrm{Y}$ & $\mathrm{Y}$ & $\mathrm{N}$ & $\mathrm{N}$ & $\mathrm{N}$ & N/A & $\mathrm{Y}$ & $\mathrm{N}$ & $\mathrm{Y}$ & $\mathrm{N}$ & N/A & $\mathrm{N}$ & POOR \\
\hline 15.Franco et al. ${ }^{36}$ & $\mathrm{Y}$ & $\mathrm{Y}$ & $\mathrm{Y}$ & $\mathrm{Y}$ & $\mathrm{Y}$ & $\mathrm{N}$ & $\mathrm{N}$ & N/A & $\mathrm{Y}$ & $\mathrm{N}$ & $\mathrm{Y}$ & $\mathrm{N}$ & N/A & $\mathrm{Y}$ & GOOD \\
\hline $\begin{array}{l}\text { 16.Magalhães et } \\
\text { al. }^{28}\end{array}$ & $\mathrm{Y}$ & $\mathrm{Y}$ & $\mathrm{Y}$ & $\mathrm{Y}$ & $\mathrm{Y}$ & $\mathrm{N}$ & $\mathrm{N}$ & N/A & Y & $\mathrm{N}$ & $\mathrm{Y}$ & $\mathrm{N}$ & N/A & $\mathrm{N}$ & REGULAR \\
\hline 17.Chow et al. ${ }^{29}$ & $\mathrm{Y}$ & $\mathrm{Y}$ & $\mathrm{Y}$ & $\mathrm{Y}$ & $\mathrm{Y}$ & $\mathrm{N}$ & $\mathrm{N}$ & N/A & $\mathrm{Y}$ & $\mathrm{N}$ & $\mathrm{Y}$ & $\mathrm{N}$ & N/A & $\mathrm{N}$ & REGULAR \\
\hline $\begin{array}{l}\text { 18.Hilger-Kolb et } \\
\text { al. }{ }^{30}\end{array}$ & $\mathrm{Y}$ & $\mathrm{Y}$ & $\mathrm{N}$ & $\mathrm{Y}$ & $\mathrm{Y}$ & $\mathrm{N}$ & $\mathrm{N}$ & N/A & $\mathrm{Y}$ & $\mathrm{N}$ & $\mathrm{Y}$ & $\mathrm{N}$ & N/A & $\mathrm{Y}$ & REGULAR \\
\hline 19.Tran et al. ${ }^{31}$ & $\mathrm{Y}$ & $\mathrm{Y}$ & $\mathrm{Y}$ & $\mathrm{Y}$ & $\mathrm{Y}$ & $\mathrm{N}$ & $\mathrm{N}$ & $\mathrm{Y}$ & $\mathrm{Y}$ & $\mathrm{N}$ & $\mathrm{Y}$ & $\mathrm{N}$ & N/A & $\mathrm{Y}$ & GOOD \\
\hline
\end{tabular}

Note: $\mathrm{n}=$ articles that met the criteria; $\mathrm{N}=$ total articles; $\%=$ percentage; $\mathrm{Y}=$ yes, attended to the item; $\mathrm{N}=$ no, did not attended to the item; N / R = not reported; N / A = not applicable.

The characteristics of the studies included in the systematic review are shown in Table 2. The articles were published between 2008 and 2018, with 12 publications between 2014 and $2018^{19-21,28-31,33-37}$. As for their impact factor, 12 articles were published in journals 18,19,21,24,26,27,29-31,33-35, with values ranging from $0.513^{33}$ to $2.567^{18}$. The type of study of all selected articles was cross-sectional (100\%). The studies were carried out in 12 different countries (Finland $^{26}$, Germany ${ }^{18,21,30}$, Bulgaria ${ }^{18,21}$, Po- land ${ }^{18,21}, \quad$ Brazil $17,19,20,25,28,32,36,37, \quad$ Sweden $^{24}$, United States ${ }^{27,31}$, Egypt $^{34}$, England ${ }^{35}$, Wales ${ }^{35}$, Northern Ireland ${ }^{35}$ and Hong Kong ${ }^{29}$ ) and one (1) study considered a sample of 26 countries from Asia, Africa and America ${ }^{33}$. The studies showed a variation in the average age of 18.4 years $^{37}$ to 24.7 years $^{36}$ and one (1) study had an amplitude of 23 to 69 years ${ }^{27}$. Regarding the courses/areas of the studied samples, most belonged to the health field, with a predominance of the Physical Education course ${ }^{17,25,32,36,37}$. 
Table 02. Characteristics of studies on self-rated health in university students.

\begin{tabular}{|c|c|c|c|c|c|c|c|}
\hline Author & $\begin{array}{c}\text { Year of } \\
\text { publication }\end{array}$ & $\begin{array}{l}\text { Impact } \\
\text { factor }\end{array}$ & Design & $\begin{array}{c}\text { Study } \\
\text { Location }\end{array}$ & Age & Gender & Courses/Area \\
\hline 1.Castrén et al. ${ }^{26}$ & 2008 & 2.067 & $\begin{array}{c}\text { Cross } \\
\text { sectional }\end{array}$ & Finland & $\begin{array}{c}19 \text { to } 35 \\
\text { years }\end{array}$ & $\mathrm{M} / \mathrm{F}$ & N/A \\
\hline $\begin{array}{l}\text { 2.Mikolajczyk } \\
\text { et al. }{ }^{18}\end{array}$ & 2008 & 2.567 & $\begin{array}{c}\text { Cross } \\
\text { sectional }\end{array}$ & $\begin{array}{l}\text { Germany, } \\
\text { Bulgaria } \\
\text { and } \\
\text { Poland }\end{array}$ & $20.7^{\star}$ & $\mathrm{M} / \mathrm{F}$ & $\begin{array}{r}\text { Natural sciences, } \\
\text { Humanities, } \\
\text { Social sciences, } \\
\text { Law and } \\
\text { Economics }\end{array}$ \\
\hline 3.Sousa ${ }^{17}$ & 2009 & $\mathrm{~N} / \mathrm{A}$ & $\begin{array}{c}\text { Cross } \\
\text { sectional }\end{array}$ & Brazil & $23.2^{*}$ & $\mathrm{M} / \mathrm{F}$ & $\begin{array}{r}\text { Physical } \\
\text { education }\end{array}$ \\
\hline 4.Sousa ${ }^{32}$ & 2010 & $\mathrm{~N} / \mathrm{A}$ & $\begin{array}{c}\text { Cross } \\
\text { sectional }\end{array}$ & Brazil & $23.2^{*}$ & $\mathrm{M} / \mathrm{F}$ & $\begin{array}{r}\text { Physical } \\
\text { education }\end{array}$ \\
\hline 5.Silva ${ }^{25}$ & 2012 & $\mathrm{~N} / \mathrm{A}$ & $\begin{array}{c}\text { Cross } \\
\text { sectional }\end{array}$ & Brazil & $\begin{array}{l}\leq 20 \\
\text { years } \\
>20 \\
\text { years }\end{array}$ & $\mathrm{M} / \mathrm{F}$ & $\begin{array}{l}\text { Physical } \\
\text { education }\end{array}$ \\
\hline 6.Schmid ${ }^{24}$ & 2012 & 1.260 & $\begin{array}{c}\text { Cross } \\
\text { sectional }\end{array}$ & Sweden & $\begin{array}{c}23.4 \\
\text { years }^{\star}\end{array}$ & $\mathrm{M} / \mathrm{F}$ & $\begin{array}{l}\text { Business } \\
\text { Administration }\end{array}$ \\
\hline 7. Rohrer et al. ${ }^{27}$ & 2012 & 1.582 & $\begin{array}{c}\text { Cross } \\
\text { sectional }\end{array}$ & $\begin{array}{l}\text { United } \\
\text { States }\end{array}$ & 23-69 & $\mathrm{M} / \mathrm{F}$ & $\mathrm{N} / \mathrm{A}$ \\
\hline 8.Freire et al. ${ }^{19}$ & 2014 & 0.780 & $\begin{array}{c}\text { Cross } \\
\text { sectional }\end{array}$ & Brazil & $\begin{array}{c}\leq 20 \\
\text { years, } \\
\text { between } \\
21 \text { and } \\
30 \text { and } \geq \\
31 \text { years. }\end{array}$ & $\mathrm{M} / \mathrm{F}$ & $\mathrm{N} / \mathrm{A}$ \\
\hline
\end{tabular}

9.Sousa et al. ${ }^{20} \quad 2014 \quad$ N/A $\quad \begin{gathered}\text { Cross } \\ \text { sectional }\end{gathered}$ Brazil $23.5^{*} \quad \mathrm{M} / \mathrm{F} \quad \begin{array}{r}\text { Health and other } \\ \text { areas }\end{array}$

\begin{tabular}{|c|c|c|c|c|c|c|c|}
\hline $\begin{array}{l}\text { 10.Pengpid et } \\
\text { al. }{ }^{33}\end{array}$ & 2015 & 0.513 & $\begin{array}{c}\text { Cross } \\
\text { sectional }\end{array}$ & $\begin{array}{c}26 \\
\text { countries } \\
\text { across } \\
\text { Asia, } \\
\text { Africa and } \\
\text { America }\end{array}$ & $20.8^{*}$ & $\mathrm{M} / \mathrm{F}$ & N/A \\
\hline
\end{tabular}

Business, Engineering, Education, Arts, Social Work, Science, Physical

\begin{tabular}{|c|c|c|c|c|c|c|c|}
\hline $\begin{array}{l}\text { 11.El-Ansari e } \\
\text { Stock }^{34}\end{array}$ & 2016 & 1.162 & $\begin{array}{c}\text { Cross } \\
\text { sectional }\end{array}$ & Egypt & $\begin{array}{c}18,9 \\
\text { years }^{*}\end{array}$ & $\mathrm{M} / \mathrm{F}$ & $\begin{array}{r}\text { Education, } \\
\text { Computer and } \\
\text { Informatics, } \\
\text { Veterinary } \\
\text { Medicine, } \\
\text { Specific } \\
\text { Education and } \\
\text { Agriculture }\end{array}$ \\
\hline
\end{tabular}




\begin{tabular}{|c|c|c|c|c|c|c|c|}
\hline $\begin{array}{l}\text { 12.Mikolajczyk } \\
\text { et al. }{ }^{21}\end{array}$ & 2016 & 2.031 & $\begin{array}{c}\text { Cross } \\
\text { sectional }\end{array}$ & $\begin{array}{c}\text { Germany } \\
\text { Bulgaria } \\
\text { Poland }\end{array}$ & $\begin{array}{c}<20 \\
\text { years, } \\
20-22 \\
\text { and }>22 \\
\text { years }\end{array}$ & $\mathrm{M} / \mathrm{F}$ & $\begin{array}{r}\text { Social sciences, } \\
\text { Natural sciences, } \\
\text { Law and } \\
\text { Economics }\end{array}$ \\
\hline $\begin{array}{l}\text { 13.El-Ansari e } \\
\text { Stock }^{35}\end{array}$ & 2016 & 1.260 & $\begin{array}{c}\text { Cross } \\
\text { sectional }\end{array}$ & $\begin{array}{l}\text { England, } \\
\text { Wales and } \\
\text { Northern } \\
\text { Ireland }\end{array}$ & N/A & $\mathrm{M} / \mathrm{F}$ & $\mathrm{N} / \mathrm{A}$ \\
\hline $\begin{array}{l}\text { 14.Andrade et } \\
\text { al. }\end{array}$ & 2017 & N/A & $\begin{array}{c}\text { Cross } \\
\text { sectional }\end{array}$ & Brazil & $18.4^{*}$ & $\mathrm{M} / \mathrm{F}$ & $\begin{array}{r}\text { Physical } \\
\text { education }\end{array}$ \\
\hline 15. Franco et al. ${ }^{36}$ & 2018 & N/A & $\begin{array}{c}\text { Cross } \\
\text { sectional }\end{array}$ & Brazil & $24.7^{\star}$ & $\mathrm{M} / \mathrm{F}$ & $\begin{array}{r}\text { Physical } \\
\text { education }\end{array}$ \\
\hline $\begin{array}{l}\text { 16.Magalhães et } \\
\text { al. }{ }^{28}\end{array}$ & 2018 & N/A & $\begin{array}{c}\text { Cross } \\
\text { sectional }\end{array}$ & Brazil & $20^{*}$ & $\mathrm{M} / \mathrm{F}$ & $\begin{array}{r}\text { Health } \\
\text { (Biomedicine, } \\
\text { Nursing, } \\
\text { Physiotherapy, } \\
\text { Medicine and } \\
\text { Occupational } \\
\text { Therapy) and } \\
\text { other areas } \\
\text { (Biological } \\
\text { Sciences, } \\
\text { Environmental } \\
\text { Engineering, } \\
\text { History, Letters, } \\
\text { Psychology and } \\
\text { Social Work) }\end{array}$ \\
\hline 17.Chow et al. ${ }^{29}$ & 2018 & 1.220 & $\begin{array}{c}\text { Cross } \\
\text { sectional }\end{array}$ & $\begin{array}{l}\text { Hong } \\
\text { Kong }\end{array}$ & N/A & $\mathrm{M} / \mathrm{F}$ & Nursing \\
\hline $\begin{array}{l}\text { 18.Hilger-Kolb } \\
\text { et al. }{ }^{30}\end{array}$ & 2018 & 2.025 & $\begin{array}{c}\text { Cross } \\
\text { sectional }\end{array}$ & Germany & $22.69^{*}$ & $\mathrm{M} / \mathrm{F}$ & $\begin{array}{r}\text { Medicine } \\
\text { Other courses }\end{array}$ \\
\hline 19.Tran et al. ${ }^{31}$ & 2018 & 1.812 & $\begin{array}{c}\text { Cross } \\
\text { sectional }\end{array}$ & $\begin{array}{l}\text { United } \\
\text { States }\end{array}$ & N/A & $\mathrm{M} / \mathrm{F}$ & N/A \\
\hline
\end{tabular}

${ }^{\star}$ Average; $\mathrm{N} / \mathrm{A}=$ Not present; $\mathrm{M}=$ Male; $\mathrm{F}=$ Female.

As for the characteristics of the studies according to the methodological criteria used (Table 3), the samples ranged from $56^{28}$ to $19,811^{33}$ participants. As a sampling criterion, there was a predominance of simple random selection ${ }^{20,25,26,28,31,33,34}$ and of selection by convenience $18,21,24,29,30,36,37$.

For the outcome assessment, different questions were observed, and the number of response options varied from four to five. The response categories used, although similar, were not exactly the same, with 13 studies using "excellent" as their first option, three studies using "very good" ("muito boa" in
Portuguese) category, one study using "very good" ("muito bom" in Portuguese) and another study using "good" as its first option. For the second response option, six studies used "good" ("boa" in Portuguese), other six studies used "very good", four studies used "good" ("bom" in Portuguese), one study used "reasonably good", and one study, "very good". For the third option, 11 studies used "regular", six studies "good" ("boa" in Portuguese) and one study, "good" ("bom" in Portuguese). In the fourth option, seven studies used "regular", nine studies "bad", one study used "quite bad", and one study used "not so 
good". Finally, for those who used five response options, nine studies used the response "bad", and four, "very bad".

Regarding the prevalence found by the HSE, 11 articles ${ }^{17,19-21,25,28,30,32,34-36}$ provided the prevalence of negative HSE outcomes dichotomously, the values varying from 3.4\%
${ }^{20}$ to $38.2 \%{ }^{28}$. In two studies ${ }^{28,34}$ the prevalence of negative HSE outcomes was greater than $30 \%$. One study, which did not consider the option "regular" as negative, had the lowest prevalence of negative HSE outcomes, $3.4 \%{ }^{20}$. The prevalence of positive HSE outcomes ranged from $61.8 \%{ }^{28}$ to $94.5 \%{ }^{21}$.

Table 03 Description of methodological criteria and prevalence of self-rated health in university students.

\begin{tabular}{|c|c|c|c|c|c|}
\hline Author & Sample & $\begin{array}{l}\text { Sampling } \\
\text { criteria }\end{array}$ & Question & $\begin{array}{c}\text { Response } \\
\text { options }\end{array}$ & Prevalence \\
\hline 1.Castrén et al. ${ }^{26}$ & 3.153 & Simple random & $\mathrm{N} / \mathrm{A}$ & $\begin{array}{l}\text { Good, Fairly } \\
\text { Good, Fair, } \\
\text { Fairly bad and } \\
\text { Bad }\end{array}$ & $\begin{array}{l}\text { Good: } \mathrm{M}: 55 \% ; \mathrm{F}=46 \% \\
\text { Fairly Good: } \mathrm{M}=31 \% ; \\
\mathrm{F}=39 \% \\
\text { Regular: } \mathrm{M}=11 \% ; \mathrm{F}= \\
14 \% \\
\text { Fairly bad: } \mathrm{M}=2 \% ; \\
\mathrm{F}=1 \% \\
\text { Bad: } \mathrm{M}=0,1 \% ; \mathrm{F}=0,2 \%\end{array}$ \\
\hline $\begin{array}{l}\text { 2.Mikolajczyk et } \\
\text { al. }{ }^{18}\end{array}$ & 2.103 & Convenience & $\begin{array}{l}\text { "How would you } \\
\text { rate your overall } \\
\text { health?" }\end{array}$ & $\begin{array}{l}\text { Excellent, } \\
\text { Very good, } \\
\text { Good, } \\
\text { Regular and } \\
\text { Bad }\end{array}$ & $\begin{array}{l}8.7 \% \text { excellent, } 35.8 \% \\
\text { very good, } 45.6 \% \text { good, } \\
8.9 \% \text { regular and } 1 \% \\
\text { poor }\end{array}$ \\
\hline 3.Sousa ${ }^{17}$ & 105 & Census & $\begin{array}{l}\text { "How do you rate } \\
\text { your current health } \\
\text { status?" } \\
\text { Question located } \\
\text { at the end of the } \\
\text { guestionnaire. }\end{array}$ & $\begin{array}{l}\text { Excellent, } \\
\text { Good, } \\
\text { Regular and } \\
\text { Bad }\end{array}$ & Negative SRH: $14.3 \%$ \\
\hline 4.Sousa ${ }^{32}$ & 105 & Census & $\begin{array}{l}\text { "How do you rate } \\
\text { your current health } \\
\text { status?" } \\
\text { Question located } \\
\text { at the beginning of } \\
\text { the questionnaire. }\end{array}$ & $\begin{array}{l}\text { Excellent, } \\
\text { Good, } \\
\text { Regular and } \\
\text { Bad }\end{array}$ & Negative SRH: $14.3 \%$ \\
\hline 5.Silva ${ }^{25}$ & 217 & Simple random & $\begin{array}{l}\text { "How do you rate } \\
\text { your current health } \\
\text { status?" }\end{array}$ & $\begin{array}{l}\text { Very Good, } \\
\text { Good, } \\
\text { Regular, Bad } \\
\text { and Very Bad }\end{array}$ & Negative SRH: 20\% \\
\hline 6.Schmid ${ }^{24}$ & 152 & Convenience & $\begin{array}{l}\text { "In general how } \\
\text { would you rate } \\
\text { your overall } \\
\text { health?" }\end{array}$ & dilu very Dad & N/A \\
\hline 7.Rohrer et al. ${ }^{27}$ & 241 & $\begin{array}{l}\text { Snowball } \\
\text { method }\end{array}$ & $\begin{array}{l}\text { "Rate your overall } \\
\text { health?" }\end{array}$ & $\begin{array}{l}\text { Excellent, } \\
\text { Very good, } \\
\text { Good, } \\
\text { Regular and } \\
\text { Poor }\end{array}$ & $\begin{array}{l}\text { Excellent, very good or } \\
\text { good SRH: } 85 \%\end{array}$ \\
\hline
\end{tabular}




\begin{tabular}{|c|c|c|c|c|c|}
\hline 8.Freire et al. ${ }^{19}$ & 874 & Conglomerates & $\begin{array}{l}\text { "How do you } \\
\text { assess your health } \\
\text { status?" }\end{array}$ & $\begin{array}{l}\text { Excellent, } \\
\text { Good, } \\
\text { Regular and } \\
\text { Bad }\end{array}$ & Negative SRH: $23.2 \%$ \\
\hline 9.Sousa et al. ${ }^{20}$ & 1.084 & Simple random & $\begin{array}{l}\text { "In general how } \\
\text { would you rate } \\
\text { your current } \\
\text { health?" }\end{array}$ & $\begin{array}{l}\text { Very Good, } \\
\text { Good, } \\
\text { Regular, Bad } \\
\text { and Very Bad }\end{array}$ & $\begin{array}{l}\text { Regular SRH: } 26.8 \% \\
\text { Negative SRH: } 3.4 \%\end{array}$ \\
\hline 10.Pengpid et al. ${ }^{33}$ & 19.811 & Simple random & $\begin{array}{l}\text { "In general, would } \\
\text { you say your health } \\
\text { is ..." }\end{array}$ & $\begin{array}{l}\text { Excellent, } \\
\text { Very good, } \\
\text { Good, } \\
\text { Regular and } \\
\text { Bad }\end{array}$ & $\begin{array}{l}\text { General classification of } \\
\text { health status: Average } \\
=3.03\end{array}$ \\
\hline $\begin{array}{l}\text { 11. El-Ansari e } \\
\text { Stock }^{34}\end{array}$ & 3.167 & Simple random & $\begin{array}{l}\text { "How would you } \\
\text { describe your } \\
\text { overall health?" }\end{array}$ & $\begin{array}{l}\text { Excellent, } \\
\text { Very good, } \\
\text { Good, } \\
\text { Regular and } \\
\text { Poor }\end{array}$ & $\begin{array}{l}\text { Excellent and very good } \\
\text { SRH: } 18.5 \% \\
\text { Good SRH: } 47.1 \% \\
\text { Regular and poor SRH: } \\
34.4 \%\end{array}$ \\
\hline $\begin{array}{l}\text { 12.Mikolajczyk } \\
\text { et al. }{ }^{21}\end{array}$ & 2.103 & Convenience & $\begin{array}{l}\text { "How would you } \\
\text { rate your overall } \\
\text { health?" }\end{array}$ & $\begin{array}{l}\text { Excellent, } \\
\text { Very good, } \\
\text { Good, } \\
\text { Regular and } \\
\text { Poor }\end{array}$ & $\begin{array}{l}\text { Positive SRH: } \\
\text { Germany: F = 90.5\% } \\
\mathrm{M}=92.5 \% \text {; Bulgaria: } \\
\mathrm{F}=92.2 \% \mathrm{M}=95.9 \% ; \\
\text { Poland: } \mathrm{F}=83.8 \% \mathrm{M}= \\
85.9 \% \\
\text { Negative SRH: } \\
\text { Germany: } \mathrm{F}=9.5 \% \mathrm{M}= \\
7.5 \% \text {; Bulgaria: } \mathrm{F}=7.8 \% \\
\mathrm{M}=4.1 \% \text {; Poland: } \mathrm{F}= \\
16.2 \% \mathrm{M}=14.1 \%\end{array}$ \\
\hline $\begin{array}{l}\text { 13. El-Ansari e } \\
\text { Stock }^{35}\end{array}$ & 3.464 & $\mathrm{~N} / \mathrm{A}$ & $\begin{array}{l}\text { "How would you } \\
\text { describe your } \\
\text { overall health?" }\end{array}$ & $\begin{array}{l}\text { Excellent, } \\
\text { Very good, } \\
\text { Good, } \\
\text { Regular and } \\
\text { Poor }\end{array}$ & $\begin{array}{l}\text { Excellent and very good } \\
\text { SRH: } 47.6 \% \\
\text { Good SRH: } 41.6 \% \\
\text { Regular and poor SRH: } \\
10.8 \%\end{array}$ \\
\hline 14.Andrade et al. ${ }^{37}$ & 84 & Convenience & $\begin{array}{l}\text { "How do you rate } \\
\text { your current health } \\
\text { status?" }\end{array}$ & $\begin{array}{l}\text { Excellent, } \\
\text { Good, } \\
\text { Regular and } \\
\text { Bad }\end{array}$ & $\begin{array}{l}\text { Excelent SRH: } 28.9 \% \\
\text { Good SRH: } 57.8 \% \\
\text { Regular SRH: } 13,3 \%\end{array}$ \\
\hline 15.Franco et al. ${ }^{36}$ & 111 & Convenience & $\mathrm{N} / \mathrm{A}$ & $\begin{array}{l}\text { Excellent, } \\
\text { Good, } \\
\text { Regular and } \\
\text { Bad }\end{array}$ & Negative SRH: 10,1\% \\
\hline $\begin{array}{l}\text { 16.Magalhães et } \\
\text { al. }{ }^{28}\end{array}$ & 56 & Simple random & $\mathrm{N} / \mathrm{A}$ & $\begin{array}{l}\text { Very good } \\
\text { to very poor } \\
\text { scale }\end{array}$ & $\begin{array}{l}\text { Very goog and good } \\
\text { SRH: } 61.8 \% \\
\text { Regular and very poor } \\
\text { SRH: } 38.2 \%\end{array}$ \\
\hline 17. Chow et al. ${ }^{29}$ & 314 & Convenience & $\mathrm{N} / \mathrm{A}$ & $\begin{array}{l}\text { Bad, Not } \\
\text { so good, } \\
\text { Regular, } \\
\text { Good and } \\
\text { Excellent }\end{array}$ & $\begin{array}{l}\text { First year students: } \\
\text { average }=3.26 \\
\text { Third year students: } \\
\text { average }=3.19\end{array}$ \\
\hline $\begin{array}{l}\text { 18.Hilger-Kolb } \\
\text { et al. }{ }^{30}\end{array}$ & 689 & Convenience & $\begin{array}{l}\text { "How do you } \\
\text { assess your general } \\
\text { health?" }\end{array}$ & $\begin{array}{l}\text { Very Good, } \\
\text { Good, } \\
\text { Regular, Bad } \\
\text { and Very Bad }\end{array}$ & Negative SRH: $13.5 \%$ \\
\hline 19. Tran et al. ${ }^{31}$ & 1.412 & Simple random & $\begin{array}{l}\text { "In general, how is } \\
\text { your health?" }\end{array}$ & $\begin{array}{l}\text { Poor, Regular, } \\
\text { Good, Very } \\
\text { Good and } \\
\text { Excellent }\end{array}$ & $\begin{array}{l}\text { Poor: } 0.57 \% \text {; Regular: } \\
\text { 5.10\%; Good: } 21.81 \% \text {; } \\
\text { Very good: } 43.41 \% \text { and } \\
\text { Excellent: } 29.11 \%\end{array}$ \\
\hline
\end{tabular}

$\mathrm{SRH}=$ self-rated health; $\%$ = Prevalence; $\mathrm{N} / \mathrm{A}=$ not present; $\mathrm{M}=$ Male; $\mathrm{F}=$ Female . 
Table 4 shows the factors associated with HSE outcomes in university students. The reviewed studies found an association for negative HSE outcomes and the following characteristics: female gender, longer course time, high stress, overweight, smoking, consuming alcohol and drugs, inadequate diet, sleep problems, inactive in leisure-time, lower economic class, health complaints, and depressive symptoms. The factors associated with a positive HSE outcome were: male gender, high level of physical activity, having high income, better mental health, normal weight, better quality of life, longer sleep duration and higher educational level of parents.

Among the statistical tests used to estimate the association (comparison) there was a predominance of the chi-square test and of the binary logistic regression. Three studies used the Poisson Regression and three other used the Multiple Linear Regression. Other studies used the Ordinal Logistic Regression, the Multinominal Logistic Regression, the Mann-Whitney test, the Kruskal-Wallis-H test, Spearman's Correlation and Fisher's Exact test.

Table 04. Factors associated with self-rated health status among university students.

\begin{tabular}{|c|c|}
\hline Author & Variables associated with the outcome \\
\hline 1.Castrén et al. ${ }^{26}$ & There was no association between variables. \\
\hline $\begin{array}{l}\text { 2.Mikolajczyk } \\
\text { et al. }^{18}\end{array}$ & $\begin{array}{c}\text { A higher score of psychosomatic complaints, }>2 \text { consultations with a doctor in the } \\
\text { last six months and insufficient income were associated with worse health status. } \\
\text { Men, a higher level of physical activity and a better sense of coherence were } \\
\text { associated with better health. }\end{array}$ \\
\hline 3.Sousa ${ }^{17}$ & $\begin{array}{l}\text { Older university women and those with a more time in the course were associated } \\
\text { with negative self-rated health. }\end{array}$ \\
\hline 4.Sousa ${ }^{32}$ & $\begin{array}{l}\text { Self-perception of negative stress and excess body weight were associated with } \\
\text { negative self-perception of health. }\end{array}$ \\
\hline 5.Silva ${ }^{25}$ & $\begin{array}{l}\text { Inadequate nutrition, problems with sleep, wearing a seat belt, stress and safe sex; } \\
\text { inappropriate behavior and introspection problems were associated with negative } \\
\text { self-rated health. }\end{array}$ \\
\hline 6.Schmid ${ }^{24}$ & $\begin{array}{l}\text { Male gender, having Swedish as a native language and mothers with high school } \\
\text { diploma were associated with better self-rated health. }\end{array}$ \\
\hline 7.Rohrer et al. ${ }^{27}$ & $\begin{array}{l}\text { Smokers, having } 14 \text { or more days of mental suffering and white students were } \\
\text { associated with lower chances of reporting a better self-rated health. }\end{array}$ \\
\hline 8.Freire et al. ${ }^{19}$ & $\begin{array}{l}\text { Female university students, those of poor economic class, insufficiently active at } \\
\text { leisure, who consume meals in restaurants, show symptoms of headache, back } \\
\text { pain and insomnia were associated with poor self-rated health (regular and bad). }\end{array}$ \\
\hline 9. Sousa et al. ${ }^{20}$ & $\begin{array}{c}\text { Negative relationships with teachers, inactive at leisure, insufficient consumption } \\
\text { of vegetables, consumption of soft drinks on most days and self-rated stress as } \\
\text { negative were associated with regular self-rated health. } \\
\text { Inactive at leisure, self-rated stress as negative and the obese were associated } \\
\text { with negative self-rated health. }\end{array}$ \\
\hline
\end{tabular}

Analysis test - Chi-square test.

- Multiple

Linear

Regression

- Chi-square test.

- Chi-square test. - Fisher's Exact test

- Poisson regression

- Multiple

Linear Regression

- Binary

Logistic

Regression

- Poisson regression

- Multinomial

Logistic

Regression 


\begin{tabular}{|c|c|c|}
\hline $\begin{array}{l}\text { 10.Pengpid et } \\
\text { al. }^{33}\end{array}$ & $\begin{array}{l}\text { Having medium or high income, having greater personal control, better mental } \\
\text { health (having no sleep problems and without or any symptoms of post-traumatic } \\
\text { stress disorder - PTSD), having normal weight, presenting inadequate eating } \\
\text { practices and a low level of activity physical activity were associated with a } \\
\text { better self-assessment of health status. }\end{array}$ & $\begin{array}{l}\text { - Multiple } \\
\text { Linear } \\
\text { Regression }\end{array}$ \\
\hline $\begin{array}{l}\text { 11. El-Ansari e } \\
\text { Stock }^{34}\end{array}$ & $\begin{array}{c}\text { University students who had a better quality of life and an awareness of their } \\
\text { health were associated with greater chances of self-rated health as very good and } \\
\text { excellent. } \\
\text { College students with higher levels of health symptoms and complaints, who } \\
\text { were so sick that they had to stay in bed, had a common cold or consulted } \\
\text { a doctor in the past } 12 \text { months, and those who felt strongly / very strongly } \\
\text { overwhelmed were associated with less chance of self-rated health as very good } \\
\text { and excellent. }\end{array}$ & $\begin{array}{l}\text { - Binary } \\
\text { Logistic } \\
\text { Regression }\end{array}$ \\
\hline $\begin{array}{l}\text { 12.Mikolajczyk } \\
\text { et al. }^{21}\end{array}$ & $\begin{array}{l}\text { Students with problems due to alcohol consumption associated with negative } \\
\text { self-rated health. }\end{array}$ & $\begin{array}{l}\text { - Binary } \\
\text { Logistic } \\
\text { Regression }\end{array}$ \\
\hline $\begin{array}{l}\text { 13. El-Ansari e } \\
\text { Stock }^{35}\end{array}$ & $\begin{array}{c}\text { Female university students, better quality of life, greater health awareness and } \\
\text { recommended levels of physical activity were associated with self-rated health as } \\
\text { very good and excellent. } \\
\text { University students with higher levels of health complaints; being sick to the } \\
\text { point of staying in bed, taking medication regularly, having seen a doctor in the } \\
\text { last } 12 \text { months, excessive alcohol consumption in the last } 2 \text { weeks and use of } \\
\text { drugs have been associated with lower chances of self-rated health as very good } \\
\text { and excellent. }\end{array}$ & $\begin{array}{l}\text { - Binary } \\
\text { Logistic } \\
\text { Regression }\end{array}$ \\
\hline $\begin{array}{l}\text { 14.Andrade et } \\
\text { al. }^{37}\end{array}$ & $\begin{array}{l}\text { College students who had a longer sleep duration and a greater amount of } \\
\text { vigorous physical activity were associated with a positive perception of health } \\
\text { (excellent). }\end{array}$ & $\begin{array}{l}\text { - Chi-square } \\
\text { test. } \\
\text { - Kruskal- } \\
\text { wallis test }\end{array}$ \\
\hline 15. Franco et al. ${ }^{36}$ & $\begin{array}{l}\text { Consumption of vegetables / salads at an irregular frequency per week, } \\
\text { overweight and negative stress were associated with negative self-rated health. }\end{array}$ & $\begin{array}{l}\text { - Poisson } \\
\text { regression }\end{array}$ \\
\hline $\begin{array}{l}\text { 16.Magalhães } \\
\text { et al. }^{28}\end{array}$ & $\begin{array}{l}\text { University students with a good to need to improve lifestyle, who showed } \\
\text { occupational behaviors of habits and involvement with the environment that need } \\
\text { to improve, were associated with the perception of regular health to very bad. } \\
\text { University students who have an excellent and very good lifestyle have } \\
\text { associated themselves with a perception of good and very good health. }\end{array}$ & $\begin{array}{l}\text { - Mann- } \\
\text { Wthiney test }\end{array}$ \\
\hline 17.Chow et al. ${ }^{29}$ & $\begin{array}{l}\text { Higher scores in health management and those who had no family conflicts in the } \\
\text { recent month were associated with positive self-rated health. }\end{array}$ & $\begin{array}{l}\text { - Ordinal } \\
\text { Logistic } \\
\text { Regression }\end{array}$ \\
\hline $\begin{array}{l}\text { 18. Hilger-Kolb } \\
\text { et al. }^{30}\end{array}$ & $\begin{array}{l}\text { The high ratio ERI (Academic Stress), high effort, low reward, and a high } \\
\text { commitment excess were associated with poor self-rated health. }\end{array}$ & Logistic \\
\hline 19. Tran et al. ${ }^{31}$ & $\begin{array}{l}\text { Student loan debt stress and depressive symptoms were associated with negative } \\
\text { self-rated health. } \\
\text { Higher family income and a higher educational level for parents were associated } \\
\text { with positive self-rated health. }\end{array}$ & $\begin{array}{l}\text { - Spearman } \\
\text { correlation }\end{array}$ \\
\hline
\end{tabular}

ERI: effort, reward, and over-commitment; BMI: body mass index.

\section{Discussion}

The present study showed that research using HSE among university students are recent, with the first selected articles published about 11 years ago ${ }^{18,26}$. There was a predominance of studies published in $2018^{28-31,36}$ and most studies were conducted in Bra$\mathrm{zil}^{17,19,20,25,26,32,36,37}$. The design of all selected articles was cross-sectional, and among the studies, differences regarding the question and answer options of HSE stood out. Inadequate nutrition, physical inactivity during leisure, high stress levels and health com- 
plaints were the factors that were most associated with negative HSE outcomes.

As for the critical assessment of the quality of the articles included in this review, there was a greater number of papers that were classified as regular. Most publications had limitations regarding the sampling aspects, with some studies with a participation rate of less than $50 \%$, while others did not justify their sample size. Factors like these increase the risk of bias, as they may not adequately represent the target population, thus leading to the question of whether there were enough participants to detect associations ${ }^{38}$. It is important to highlight that, from the 19 studies, 12 were published in journals with an impact factor from 0.513 to 2.567 , numbers considered as representative in the science field. But this can point to the fact that elements inherent to methodological procedures may not have been judged by the evaluators as limiting factors in publications.

Regarding HSE, since it is a self-reported measure, the use of questionnaires is the only way to obtain information. The questionnaire has been one of the most used information extraction instruments due to its practicality and low application $\operatorname{cost}^{39}$. However, in this study, the characteristics of the question, answer options, and the categorization of the variables were not standardized, which makes it difficult to compare the surveys. Thus, the variation in the prevalence of negative HSE, that range from $3.4 \%^{20}$ to $38.2 \%{ }^{28}$, mainly due to the classification criteria. In certain studies, the options "regular" and "bad" were considered as a negative HSE, while in others, the options "regular", "bad" and "very bad" or "bad" and "very bad" were considered as negative.

This review found that articles consistently included the "regular" option as a negative assessment. This was also observed in a systematic review of HSE in the elderly, which showed a prevalence of negative assessments greater than $30 \%$ in the surveys that included the responses "regular"

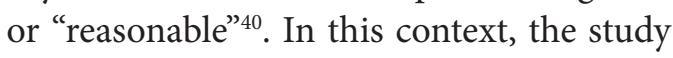
of Sousa et al..$^{20}$, included in this review, did not consider "regular" as negative response, and observed a lower prevalence of negative HSE (3.4\%). This result is close to the data of the VIGITEL study with adults from Brazilian capitals performed in 2018, where a negative assessment of their health status (as bad or very bad) was of $4.6 \%{ }^{41}$. However, there are specific observations of regular HSE in university students ${ }^{20}$, since it was noted that health behaviors were more associated with this option. It is possible to suggest that research analyze the intermediate categories grouped with the categories that estimate the negative health level, since there is a mortality risk consideration for people who perceive health as regular ${ }^{42}$.

Among the factors associated with HSE, there was a greater number of publications in this review that showed the relationship between stress and health complaints with negative HSE. The association of these attributes was observed in studies with adolescents, which showed the association of negative stress with negative $\mathrm{HSE}^{43}$. In research with adult industry workers, it was observed that patients with limiting diseases and who needed longer short-term leave had higher chances of negatively assessing health ${ }^{44}$. These two cha- 
racteristics (stress and health complaints) can be related to each other, as shown in a study with university students from the United Kingdom and Egypt ${ }^{45}$ and adolescents from Canada that revealed the negatively physical and mental status were associated with quality of life, on the other hand global quality of life was more determined by the mental health status ${ }^{46}$. However, in this review, the studies did not investigate these characteristics simultaneously.

The causes of stress and health complaints need to be better clarified through investigations regarding time spent at university, environment, emotional challenges, and decreased family relationships. This would allow to better understand the coping mechanisms of psychosocial risks among university students.

In addition, it was observed that inadequate diet and being overweight were directly associated with negative HSE outcomes in university students. The association of these variables was also verified in a systematic review with adolescents ${ }^{47}$. College students who adopt unhealthy eating habits are more susceptible to the development of chronic non-communicable diseases, such as obesity. Thus, the recognition of the negative role of these behaviors can increase the likelihood of a negative self-evaluation.

Physical activity is a behavior that, in this study, was sometimes associated with the HSE outcomes in a negative way, and sometimes, in a positive one. In studies in which university students reached the recommended levels of physical activity ${ }^{35}$ and in those in which they reached a higher level of physical activity ${ }^{18,37}$ there was an association with positive HSE outcomes. However, in studies with university students that did not reach the recommended level of physical activity at leisure ${ }^{19}$ or were physically inactive at leisure ${ }^{20}$, an association with negative HSE outcomes was observed. This corroborates the review conducted by Vancea et $\mathrm{al} .{ }^{48}$ with the adolescent population, which showed that, among selected studies, there was evidence of a direct association between the practice of physical activities and a positive perception of health. In the review conduct by Silva et al. ${ }^{47}$, among the factors associated with negative HSE outcomes in adolescents, low levels of physical activity were also presented as a strong indicator. This aspect can be explained by the physiological and psychosocial effects that physical activity generates in HSE.

In this review, the study of Andrade et al. ${ }^{37}$ only showed an association of greater amounts of physical activity at vigorous intensity with positive HSE, whereas when it comes to moderate intensity this association was not observed. This result may have been influenced by the study sample (86 university students), and since there was no justification for this number, the power of the sample for estimating results may have been neglected, since small samples may be associated with larger errors ${ }^{38}$. Another aspect of this publication was the lack of adjustment for potential confounding variables, which adds possible biases to the results. On the other hand, it was found that, out of the 19 studies, 13 performed robust analyzes through regressions, which allow inferences based on control models for confounding factors ${ }^{49}$, and thus makes it possible to estimate the determinants of HSE in university students.

In relation to the analysis of socioeconomic factors, higher family income was 
shown to be associated with positive HSE. Review studies focusing on adolescents ${ }^{47}$ and elderly ${ }^{40}$ demonstrated the same result. The level of income reflects the possibility of acquiring good food, greater access to leisure activities, education, housing, and health services. However, these results must be analyzed carefully. A systematic review with longitudinal studies on the relationship between income and health showed that the association of positive HSE with higher income is very small and probably insignificant when the result is adjusted for confounding factors, such as employment status, social support, and family structure ${ }^{45}$.

There was a lack of longitudinal studies on this subject in university students. Longitudinal data are considered to have greater explanatory power. A cross-sectional study has the impossibility to establish cause and effect relationships ${ }^{50}$. Another aspect verified in this review was that most of the studies were carried out with samples of students from a specific course ${ }^{17,24,25,29,32,36,37}$. This can lead to bias in relation to the generalization of information for the general population of university students.

In this review, the limitations refer to the non-inclusion of publications such as theses and dissertations, which could expand the information regarding the theme. As a strength of this study, the main databases were used to expand the search strategy and studies from different countries and years of publication were included, which allows characterizing HSE in university students.

In view of the findings of this systematic review, due to the wide amplitude of prevalence, other research is suggested that incorporate the validation of a question and a standardized scale to be used to estimate
HSE, especially considering the balance between the negative and positive poles, and the intermediate option. This review is one of the first to systematize studies on HSE in the university population.

\section{Conclusion}

This review showed a high amplitude in the prevalence of negative HSE in university students from different countries. As for the methodological aspects, the lower quality of the studies was related to their sampling aspects. In addition, it was found that the options for answering the question about HSE are not standardized, which makes it difficult to compare results. There are a wide range of variable that can determine HSE, like sociodemographic, psychosocial, behavioral, lifestyle, and quality of life factors. Thus, it is important to monitor indicators in university spaces, helping in decision making regarding health policies for university students.

\section{ACKNOWLedgments}

To the Postgraduate Program in Physical Education at the Federal University of Triângulo Mineiro, as this study is part of the dissertation entitled "Negative Health Self-Assessment in University Students: analysis in relation to physical activity, sedentary time and hours of sleep", presented to the program. The first author would like to thank the Coordination for the Improvement of Higher Education Personnel (CAPES) for the financial support as a scholarship at the Master's level and the Municipal Education Department (SEMEC) - Teresina/PI, for the authorization of the leave to attend continuing education. 


\section{REFERENCES}

1. Peres MA, Masiero AV, Longo GZ, Rocha GC, Matos IB, Najnie K, et al. Auto-avaliação da saúde em adultos no Sul do Brasil. Rev Saúde Públ 2010; 44(5):901-11.

2. Lima-Costa MF, Cesar CC, Chor D, Proietti FA. Self-rated health compared with objectively measured health status as a tool for mortality risk screening in older adults: 10-year follow-up of the Bambuí Cohort Study of Aging. Am J Epidemiol. 2012; 175(3): 228-35.

3. World Health Organization (WHO). Health interview surveys: towards international harmonization of methods and instruments. Disponível: http://apps.who.int/iris/handle/10665/107328

4. Hodačová L, Hlaváčková E, Sigmundová D, Kalman M, Kopčáková J. Trends in Life Satisfaction and Self-rated Health in Czech School-aged Children: HBSC Study. Cent Eur J Public Health. 2017; 25(1):51-6.

5. Malta DC, Oliveira MM, Machado IE, Prado RR, Stopa SR, Crespo CD, et al. Characteristics associated to a poor self-rated health in Brazilian adolescents, National Adolescent School-based Health Survey, 2015. Rev Bras Epidemiol. 2018; 21(1):e180018.

6. Liu F, Zhang C, Liang Y, Deng Q, Hang D, Pan Y, et al. Epidemiology of self-rated health in rural China: a population-based cross-sectional study. Sci Rep. 2017; 7(1): 4459.

7. Abuladze L, Kunder N, Lang K, Vaask S. Associations between self-rated health and health behaviour among older adults in Estonia: a cross-sectional analysis. BMJ Open. 2017;7(6):e013257.

8. Feng Q, Zhu H, Zhen Z, Gu D. Self-Rated Health, Interviewer-Rated Health, and Their Predictive Powers on Mortality in Old Age. J Gerontol B Psychol Sci Soc Sci. 2016; 71(3):538-50.

9. Moreno X, Huerta M, Albala C. Autopercepción de salud general y mortalidad en adultos mayores. Gac Sanit. 2014;28(3):246-52.

10. Pavão ALB, Werneck GL, Campos MR. Autoavaliação do estado de saúde e a associação com fatores sociodemográficos, hábitos de vida e morbidade na população: um inquérito nacional. Cad Saúde Pública. 2013; 29(4):723-34

11. Andrade GF, Loch MR, Silva AMR, Andrade GF, Loch MR, Silva AMR. Mudanças de comportamentos relacionados à saúde como preditores de mudanças na autopercepção de saúde: estudo longitudinal (2011-2015). Cad Saúde Pública 2019; 35(4): e0015141.

12. Kananen L, Enroth L, Raitanen J, Jylhävä J, Bürkle A, Moreno-Villanueva M et al. Self-Rated Health in Individuals with and without Disease Is Associated with Multiple Biomarkers Representing Multiple Biological Domains. Sci Rep. 2021;11(1):6139.

13. Tyrer P. The Behavior of Academics Under Stress: A Commentary on Blashfield and Reynolds. J Pers Disord 2012; 26(6):835-7.

14. Brandão MP, Pimentel FL, Cardoso MF. Impact of academic exposure on health status of university students. Rev Saúde Públ. 2011; 45(1):49-58.

15. Kwan MY, Cairney J, Faulkner GE, Pullenayegum EE. Physical activity and other health-risk behaviors during the transition into early adulthood: a longitudinal cohort study. Am J Prev Med. 2012;42(1):14-20.

16. Sousa TF, José HPM, Barbosa AR. Condutas negativas à saúde em estudantes universitários brasileiros. Cien Saude Colet. 2013;18(12):3563-75.

17. Sousa TF. Auto-Avaliação do Nível de Saúde em Estudantes de Educação Física. Saúde e Pesquisa 2009; 2(1):17-21.

18. Mikolajczyk RT, Brzoska P, Maier C, Ottova V, Meier S, Dudziak U, et al. Factors associated with self-rated health status in university students: a cross-sectional study in three European countries. BMC Public Health 2008; 8:215. 
19. Freire LM, Dalamaria T, Cunha MA, Souza OF. Self-rated health in university students from Rio Branco in the Western Brazilian Amazon. Health. 2014; 6(16):2245-9.

20. Sousa T, Fonseca SA, Barbosa AR. Regular and negative self-rated health in students from a public university from Northeastern, Brazil: prevalence and associated factors. Acta Sci Anim Sci. 2014;36(2):185-94.

21. Mikolajczyk RT, Sebena R, Warich J, Naydenova V, Dudziak U, Orosova O. Alcohol Drinking in University Students Matters for Their Self-Rated Health Status: A Cross-sectional Study in Three European Countries. Front Public Health. 2016; 4:210.

22. Liberati A, Altman DG, Tetzlaff J, Mulrow C, Gøtzsche PC, Ioannidis JPA, et al. The PRISMA statement for reporting systematic reviews and meta-analyses of studies that evaluate healthcare interventions: explanation and elaboration. BMJ 2009; 6(7):1-28.

23. Assessing Cardiovascular Risk: Systematic Evidence Review from the Risk Assessment Work Group | National Heart, Lung, and Blood Institute (NHLBI) Disponível em: https://www.nhlbi. nih.gov/health-topics/assessing-cardiovascular-risk

24. Schmidt M. Predictors of Self-rated Health and Lifestyle Behaviours in Swedish University Students. Glob J Health Sci. 2012; 4(4):1-14.

25. Silva DAS. Indicadores do estilo de vida e autoavaliação negativa de saúde em universitários de uma instituição pública do nordeste do Brasil. Rev Bras Ativ Fís Saúde. 2012; 17(4):263-9.

26. Castrén J, Huttunen T, Kunttu K. Users and non-users of web-based health advice service among Finnish university students - chronic conditions and self-reported health status (a cross-sectional study). BMC Med Inform Decis Mak. 2008; 8(1):1-8.

27. Rohrer JE, Cole LJ, Schulze FW. Cigarettes and Self-Rated Health Among Online University Students. J Immigr Minor Health 2012; 14(3):502-5.

28. Magalhães BC, Vieira PM, Aramaki AL, Kososki E, Dutra FCMS. Percepción de la salud, estilo de vida y el comportamiento ocupacional de los estudiantes universitarios. Rev Chil Ter Ocup 2018; 18(1):17-26.

29. Chow SKY, Lam K-M, Lie S-H, Mak K-C, Mong K-C, So C-M, et al. Do demographic factors and a health-promoting lifestyle influence the self-rated health of college nursing students? BMC Nurs 2018; 17:50.

30. Hilger-Kolb J, Diehl K, Herr R, Loerbroks A. Effort-reward imbalance among students at German universities: associations with self-rated health and mental health. Int Arch Occup Environ Health 2018; 91(8):1011-20.

31. Tran AGTT, Mintert JS, Llamas JD, Lam CK. At what costs? Student loan debt, debt stress, and racially/ethnically diverse college students' perceived health. Cultur Divers Ethnic Minor Psychol. 2018; 24(4):459-69.

32. Sousa TF. Autopercepção negativa de saúde e fatores associados em acadêmicos de Educação Física no Nordeste, Bahia. Rev Digital 2010; 15(143).

33. Pengpid S, Peltzer K, Samuels TA, Gasparishvili A. Factors associated with self-rated health status among university students from 26 low, middle and high income countries. Journal of Psychology in Africa. 2015; 25(5):448-53.

34. El Ansari W, Stock C. Explaining the gender difference in self-rated health among university students in Egypt. Women Health. 2016; 56(7), 731-744.

35. El Ansari W, Stock C. Gender Differences in Self-Rated Health among University Students in England, Wales and Northern Ireland: Do Confounding Variables Matter? Glob J Health Sci. 2016; 8(11):168-77.

36. Franco DC, Dias MS, Sousa TF. Percepção negativa de saúde e fatores associados em acadêmicos de Educação Física. ABCS Health Sci. 2018; 43(3):163-8. 
37. Andrade RD, Felden ÉPG, Teixeira CS, Pelegrinil A. Sono, percepção de saúde e atividade física em adolescentes universitários. Adolesc Saude. 2017; 14(4):150-6.

38. Luiz RR, Magnanini MMF. A lógica da determinação do tamanho da amostra em investigações epidemiológicas. Cad. saúde coletiva. 2000;8(2):9-28.

39. Freitas AL, Rodrigues $\mathrm{S}$. A avaliação da confiabilidade de questionários: uma análise utilizando o coeficiente alfa de Cronbach. In: XII SIMPEP, 2005, Bauru, SP, Brasil.

40. Pagotto V, Bachion MM, Silveira EA. Autoavaliação da saúde por idosos brasileiros: revisão sistemática da literatura. Rev. Panam. Salud Publica. 2013; 33(4):302-10.

41. Brasil. Ministério da Saúde. VIGITEL Brasil, 2018: Vigilância de Fatores de Risco e Proteção para Doenças Crônicas por Inquérito Telefônico: estimativas sobre frequência e distribuição sócio-demográfica de fatores de risco e proteção para doenças crônicas nas capitais dos 26 Estados brasileiros e no Distrito Federal em 2018. Brasília: Ministério da Saúde; 2019. Disponível: https://portalarquivos2.saude.gov.br/images/pdf/2019/julho/25/vigitel-brasil 2018.pdf

42. Bopp M, Braun J, Gutzwiller F, Faeh D. Health Risk or Resource? Gradual and Independent Association between Self-Rated Health and Mortality Persists Over 30 Years. PlosOne 2012; 7(2): e30795.

43. Sousa TF, Silva KS da, Garcia LMT, Del Duca GF, Oliveira ESA, Nahas MV. Autoavaliação de saúde e fatores associados em adolescentes do Estado de Santa Catarina, Brasil. Rev Paul Pediatr. 201028(4):333-9.

44. Höfelmann DA, Blank N. Auto-avaliação de saúde entre trabalhadores de uma indústria no sul do Brasil. Rev Saúde Públ. 2007; 41(5):777-87.

45. Reche E, König H-H, Hajek A. Income, Self-Rated Health, and Morbidity. A Systematic Review of Longitudinal Studies. Int J Environ Res Public Health. 2019; 16(16):2884.

46. Sawatzky R, Ratner PA, Johnson JL, Kopec JA, Zumbo BD. Self-reported physical and mental health status and quality of life in adolescents: a latent variable mediation model. Health Qual Life Outcomes. 2010; 8:17

47. Silva BVS, Andrade PMC, Baad VMA, Valença PAM, Menezes VA, Amorim VC, et al. Prevalência e fatores associados à autopercepção negativa em saúde dos adolescentes: uma revisão sistemática. Rev Bras Promoç Saúde. 2016; 29(4):595-601.

48. Vancea LA, Barbosa JMV, Menezes AS, Santos CM, Barros MVG. Associação entre atividade física e percepção de saúde em adolescentes: Revisão Sistemática. Rev Bras Ativ Fís Saúde. 2011; 16(3):246-54.

49. Coutinho LMS, Scazufca M, Menezes PR. Métodos para estimar razão de prevalência em estudos de corte transversal. Rev Saúde Pública. 2008; 42(6):992-8.

50. Sitta ÉI, Arakawa AM, Caldana ML, Peres SHCS. A contribuição de estudos transversais na área da linguagem com enfoque em afasia. Rev. CEFAC. 2010; 12(6):1059-66.

\section{DAdOS DOS AUTORES}

\section{Mariana da Silva Ferreira}

Professora de Educação física atuante na Secretaria Municipal de Educação de Teresina (SEMEC), Teresina/PI - Brasil. marianaferreira_83@hotmail.com 


\section{Gildeene Silva Farias}

Professor do curso de bacharelado em educação física na Faculdade Estácio de Teresina, Teresina/PI - Brasil. gildeene83@gmail.com

\section{Sandra Adriana Neves Nunes}

Professora do ensino superior na Universidade Federal do Sul da Bahia (UFSB), Itabuna/ BA - Brasil.sandranunes@ufsb.edu.br

\section{Camila Bosquiero Papini}

Professora adjunta do departamento de ciências do esporte na Universidade Federal do Triângulo Mineiro (UFTM), Uberaba/MG - Brasil. camila.papini@uftm.edu.br

\section{Thiago Ferreira de Sousa}

Professor do ensino superior na Universidade Federal do Recôncavo da Bahia (UFRB), Amargosa/ BA - Brasil - Brasil. tfsousa_thiago@yahoo.com.br

Submetido em: 15-5-2021

Aceito em: 11-10-2021 